\title{
Does Lack of EWSR1-ATF1 Fusion Exclude Diagnosis of Salivary Gland Clear Cell Carcinoma?
}

\author{
Sibel Sensu ${ }^{1 *}$, Nusrat Erdogan ${ }^{2}$, Kayhan Basak ${ }^{2}$, Egzona Qipa ${ }^{3}$, Murat Byukdogan ${ }^{4}$ \\ ${ }^{1}$ Pathology Department, Medical Faculty, Istinye University, Istanbul, Turkey \\ ${ }^{2}$ Clinical Pathology Department, University of Health Sciences Kartal Dr. Lutfi Kirdar Education and Research Hospital, \\ Istanbul, Turkey \\ ${ }^{3}$ Medical Genetics Department, Istinye University, Istanbul, Turkey \\ ${ }^{4}$ Genetics Diagnosis Center, DONEGEN, Istanbul, Turkey
}

\section{ARTICLE INFO}

Received : 29 October 2020

Reviewed : 24 January 2021

Accepted : 13 April 2021

Keywords:

clear cell carcinoma, ESWR1-ATF1, immunohistochemistry, salivary gland

*Corresponding author:

\section{Sibel Sensu}

Pathology Department, Medical Faculty,

Istinye University, Istanbul, Turkey

ssensu@yahoo.com

\begin{abstract}
Introduction: Ewing sarcoma breakpoint region 1- activating transcription factor 1 (EWSR1-ATF1) fusion is mentioned as a diagnostic marker in favor of clear cell carcinoma in the differential diagnosis of clear cell neoplasms of salivary glands. However, in literature, its positivity is reported between $82 \%$ and $93 \%$. In salivary glands, various benign and malignant primary tumors, as well as odontogenic neoplasms and metastatic cases, present with a varied number of clear cells. Clear cell carcinoma of the salivary gland is a rare entity with distinct histochemistry and immunohistochemistry features.
\end{abstract}

Case Presentation: We present a clear cell carcinoma of the salivary gland showing cytoplasmic Periodic acid-Schiff (PAS) (+), mucin (-) staining, cytokeratin (CK), CK7, and P63 immunoreactivity without EWSR1-ATF1 fusion.

Conclusions: We conclude that clinical, morphological, and immunohistochemical analyses should be the guide for diagnosis even though molecular analysis does not support it.

\section{INTRODUCTION}

Salivary gland tumors constitute a very heterogeneous group that accounts for approximately $2-6.5 \%$ of head and neck neoplasms [1]. They consist of tumors with distinct clinical and histological characteristics and diverse biological behaviors with five-year survival between 25\% and over 95\% [1-3]. Recently, Ewing sarcoma breakpoint region 1-activating transcription factor 1 (EWSR1-ATF1) fusion was found as a marker specific to salivary gland clear cell carcinomas $[4,5]$. Here, we discuss the value of ESWR1-ATF1 fusion in the diagnosis of a salivary gland tumor with clear cells.

\section{CASE PRESENTATION}

A forty-eight-year-old male patient was admitted due to a mass of three-year duration on his right cheek. Physical examination revealed a $3 \times 3-\mathrm{cm}$ nodular, firm, subcutaneous lesion that involved the cheek mucosa. The tumor was removed by excisional biopsy in several fragments. Microscopically, the lesion was composed of an epithelial island of tumor cells with small central nuclei and abundant clear cytoplasm (Figure 1) within salivary gland tissue without prominent atypia, mitotic activity, necrosis, or hyalinization. Tumor cells were Periodic acid-Schiff (PAS) (+), Diastase PAS (DPAS) and mucin (-) and immunohistochemically, cytokeratin (CK) (+), P63 (+) (Figure 2), CK 7, epithelial membrane antigen (EMA) and carcinoembryonic antigen (CEA) focal $(+)$, Ki67 1\% (+), S-100, smooth muscle actin (SMA), discovered on GIST-1 (DOG1), Vimentin, CK20, Paired box (PAX)2, PAX8, and sex-determining region Y-box 10 (SOX10) (-). Molecular investigation for EWSR1 with fluorescent in situ hybridization (FISH) analysis did not reveal a positive signal and was reported as an absence of fusion (Figure 3). Results of neck ultrasound, upper/ lower abdomen, and thorax computerized tomography (CT), as well as the examination of both kidneys, were normal. Altogether, the diagnosis was clear cell carcinoma of the salivary gland. 


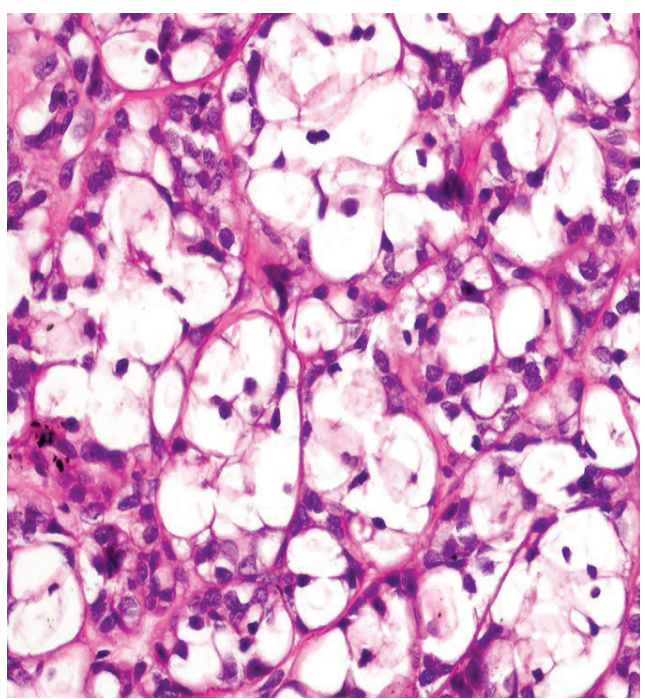

Figure 1. Tumor cells with central small nuclei and large clear cytoplasm (H\&E, magnification 400x).

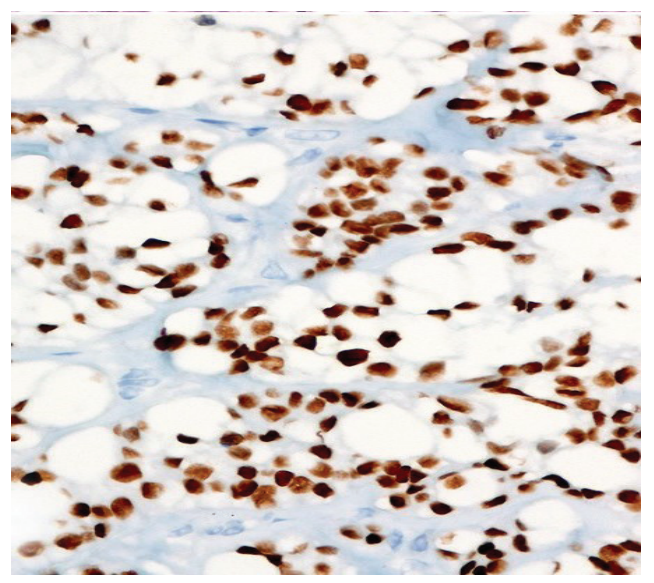

Figure 2. Nuclear P63 immunoreactivity (P63, magnification 400x).

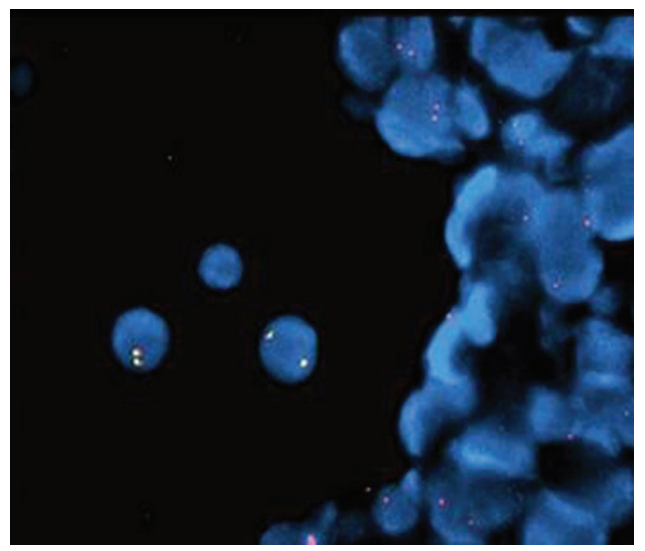

Figure 3. Negative break-apart fluorescence in-situ hybridization analysis for EWSR1 showing tumor cells with two normal fusion signals

Due to the removal of the lesion in fragments and suspicion of residual cancer, wide excision/right neck dissection was performed after two months, and the final report revealed clear cell carcinoma with negative surgical margins and no lymph node involvement. The patient is still under regular follow-up for over three years without any tumor-based problems.

\section{DISCUSSION}

Several benign and malignant tumors of salivary glands, besides metastatic or odontogenic entities, should be considered in the differential diagnosis. Primary tumors, such as mucoepidermoid carcinoma and clear cell carcinoma, metastases from renal cell carcinoma (RCC) and malignant melanoma, and clear cell tumor of odontogenic origin should be taken into account [2-6]. Clear cell carcinoma (CCC), which accounts for less than $1 \%$ of all salivary gland neoplasms, has a low-grade morphology, very low metastatic potential, and a good prognosis. EWSR1 gene rearrangements found in $45 \%$ of soft tissue myoepithelial tumors (SMET) are also identified in most of the clear cell carcinomas of salivary glands $[4,5]$. EWSR1 gene might have a role in the oncogenesis of various soft tissue, skin, and salivary gland tumors. EWSR1 translocations have been detected in many entities such as Ewing sarcoma/peripheral neuroectodermal tumor, desmoplastic small round cell tumor, clear cell sarcoma of soft tissue, angiomatoid fibrous histiocytoma, extraskeletal myxoid chondrosarcoma, myxoid liposarcoma, soft tissue myoepithelioma, and clear cell hidradenoma [3], and some of these entities might have morphological similarities to CCC. SMET is an S100 and glial fibrillary acidic protein (GFAP) positive tumor group. Aiming to clarify a possible link between CCC and myoepithelial tumors, Shah et al. [7] found EWSR1 rearrangement in $87 \%$ of CCCs; however, CCC was negative for S100, GFAP, and all myoid markers. Contrary to SMET that showed POU class 5 homeobox 1 (POU5F1) rearrangement in addition to EWSR1, any EWSR1POU5F1 fusion was not found in a series of 23 cases of CCC. Therefore, a relation could not be established between these two entities.

In literature, EWSR1 gene rearrangement rates are reported between $66 \%-87 \%$, and an ATF1 rearrangement is reported in $93 \%$ of CCC cases [7-9]. A high (up to $33 \%$ ) rate of EWSR1-split negativity in various reports, as observed in our case, might be an indication of an etiology other than an EWSR1 gene abnormality in some cases. On the other hand, EWSR1 gene is shown to have distinct fusions with various genes in different neoplasms. CREB1 and CREB3L2 are two genes that belong to the CAMP response element-binding protein (CREB) transcription factor gene family. EWSR1-ATF/CREB gene fusions can also be observed as very early events in tumorigenesis. Some other genetic or epigenetic events might be responsible for particular phenotypes [10]. Thus, in required cases, EWSR1- ATF1/CREB1 analysis might be investigated to support pathological evaluation. Also, examination with reverse transcription- 
polymerase chain reaction (RT-PCR) and direct sequencing might be added, if necessary.

On the other hand, in a series of 94 salivary gland carcinomas, $39 \%$ of the cases of clear cell myoepithelial carcinomas ( $\mathrm{p} 63$ and S100 positive) showed ESWR1 gene rearrangement [11]. Also, the same fusion might be detected in clear cell sarcoma, gastrointestinal clear cell sarcomas like tumors and certain angiomatoid fibrous histiocytomas while not detected in some of the CCC cases $[5,8]$. Our case, like some reports in the literature [7-9], did not show an EWSR1 mutation, but morphological, histochemical, and immunohistochemical findings supported CCC.

Tumor cells of salivary gland CCC are pancytokeratin positive with, in almost all cases, a strong and diffuse P63 positivity. Mostly, focal or diffuse EMA, CK7, CK19, and CAM5.2 immunoreactivity are found. In CCC, S100, SMA, and calponin staining supporting myoepithelial differentiation are not observed. Also, tumor cells are muscle-specific actin (MSA), GFAP, CK8, CD19 (in favor of metastasis), and vimentin negative $[8,12]$. In differential diagnosis, PAX2 and PAX8 positivity are in favor of metastatic RCC; SOX10, malignant melanoma, and CK7(+)/CK20(-) immunostaining, in favor of primary salivary gland tumor. Immunostaining is not helpful for the differentiation of CCC from clear cell odontogenic carcinoma. Our case showed immunohistochemical staining that excluded metastatic entities such as RCC and malignant melanoma (CK7 positive; CK20, PAX2, PAX8, SOX10 negative) and supported a primary salivary gland malignancy (CK, P63 positive) $[2-4,8]$.

\section{CONCLUSIONS}

Generally, for malignant salivary gland tumor composing of clear cells, clear cell carcinoma of the salivary gland, clear cell-rich mucoepidermoid carcinoma as well as odontogenic and metastatic tumors, particularly RCC should be considered. Our case was diagnosed as clear cell carcinoma of the salivary gland due to clinical and morphological features; PAS (+), DPAS, and mucin (-) staining; CK, CK7, and P63 immunoreactivity. However, EWSR1 gene mutation reported in most of the CCCS was not detected. Primary salivary gland CCC is diagnosed by clinical, morphological, histochemical, and immunohistochemical examination through ESWR1-ATF1 fusion, which might be helpful in some problematic cases. The prognostic significance of subgroups with and without ESWR1-ATF1 fusions should be investigated.

\section{DECLARATIONS}

\section{Competing of Interest}

The authors declare no competing interest in this study.

\section{Ethics Approval}

All diagnostic and therapeutic procedures were performed after informed consent of the patient and Istinye University Ethics Committee Approval was obtained (Ethics Committee Approval Nr: 07/06.01.2020).

\section{Acknowledgment}

None.

\section{REFERENCES}

1. Ant A, Toprak F, Duran A, et al. The demographic and clinicopathological features of salivary gland tumors in Turkey. Ortadogu Tip Derg. 2019;11(4):450-5.

2. Bhamjee $F$, Jeftha $A$, Holmes $H$, et al. Hyalinising clear cell carcinoma of the maxilla in a young adult female. SADJ. 2018;73(2):86-8.

3. Devaraju RR, Reddy S, Yashoda, et al. Clear cell odontogenic carcinoma versus hyalinizing clear cell carcinoma: A diagnostic dilemma. J Indian Acad Oral Med. 2014;26(3):319-22.

4. Albergotti WG, Bilodeau EA, Byrd JK, et al. Hyalinizing clear cell carcinoma of the head and neck: Case series and update. Head Neck. 2016;38:426-33.

5. Seethala RR, Stenman G. Update from the 4th Edition of the World Health Organization Classification of Head and Neck Tumours: Tumors of the Salivary Gland. Head and Neck Pathol. 2017;11(1):55-67.

6. Yang $\mathrm{XH}$, Liu L, Shi YY, et al. Hyalinizing clear cell carcinoma of salivary gland origin in the head and neck: clinical and histopathological analysis. Int J Oral Maxillofac Surg. 2018;47:692-8.

7. Shah AA, LeGallo RD, Zante A, et al. EWSR1 genetic rearrangements in salivary gland tumors. a specific and very common feature of hyalinizing clear cell carcinoma. Am J Surg Pathol. 2013;37:571-8.

8. Weinreb I. Hyalinizing clear cell carcinoma of salivary gland: a review and update. Head and Neck Pathol. 2013;7:S20-S29.

9. Nakano $\mathrm{T}$, Yamamoto $\mathrm{H}$, Nishijima $\mathrm{T}$, et al. Hyalinizing clear cell carcinoma with EWSR1-ATF1 fusion gene: report of three cases with molecular analyses. Virchows Arch. 2015;466(1):37-43.

10. Vogels R, Baumhoer D, van Gorp J, et al. Clear cell odontogenic carcinoma: occurrence of EWSR1-CREB1 as alternative fusion gene to EWSR1-ATF1. Head Neck Pathol. 2019;13(2):225-30.

11. Skalova A, Weinreb I, Hyrzca M, et al. Clear cell myoepithelial carcinoma of salivary glands showing EWSR1 rearrangement: Molecular analysis of 94 salivary gland carcinomas with prominent clear cell component. Am J Surg Pathol. 2015;39(3):338-48.

12. Sanjai K, Shivalingaiah D, Sharath R, Pandey B. Clear cell carcinoma of palatine salivary gland: A diagnostic challenge. J Oral Maxillofac Pathol. 2018;22:128-31. 